\title{
A prospective study of thyroid function, morphology and autoimmunity in young patients with type 1 diabetes
}

\author{
D Hansen, F N Bennedbæk ${ }^{1}$, M Høier-Madsen ${ }^{2}$, L Hegedüs ${ }^{1}$ and B B Jacobsen \\ Department of Paediatrics and ${ }^{1}$ Department of Endocrinology, Odense University Hospital, 5000 Odense C, Denmark and \\ ${ }^{2}$ Department of Autoimmunology, State Serum Institute, Copenhagen, Denmark
}

(Correspondence should be addressed to Dorte Hansen; Email: dorte.hansen@dadlnet.dk)

\begin{abstract}
Objective: Thyroid autoantibodies (TA) and thyroid ultrasonography are widely used in the diagnosis of autoimmune thyroid disease (AITD). However, we know little of the significance of having ultrasonographic abnormalities (USabn) without having any other signs of AITD. In a previous populationbased study of 105 young patients with type 1 diabetes (T1DM) we found a high prevalence (42\%) of USabn. In the present study we evaluate the development of both USabn and TA in a 3-year follow-up of this cohort.

Design: Of the 105 previously investigated children and adolescents with T1DM (aged 5-21 years), 101 were re-examined. Serum concentrations of tri-iodothyronine $\left(\mathrm{T}_{3}\right)$, thyroxine $\left(\mathrm{T}_{4}\right)$, TSH, thyroid peroxidase antibodies (TPOab) and thyroglobulin antibodies (Tgab), as well as thyroid size and morphology were determined in all patients.

Results: During the 3 years follow-up period, the prevalence of thyroid dysfunction increased from 5 to $8 \%$, the prevalence of TPOab was unchanged at 13\%, while the prevalence of Tgab decreased from 14 to $7 \%$. The prevalence of USabn increased from 42 to $49 \%$. Most patients presented USabn at both examinations. Patients with USabn had a higher prevalence of TA than those without USabn $(P=0.038)$ and higher serum levels of TSH $(P=0.027)$. All patients with thyroid dysfunction presented with USabn. However, many patients with USabn had no other signs of AITD.

Conclusions: A high prevalence of thyroid dysfunction, TA and thyroid USabn were found in young patients with T1DM. Thyroid USabn was a sensitive but non-specific marker of AITD and is therefore unsuitable for screening purposes. Instead, we recommend regular screening using serum TSH in the follow-up of young diabetic patients.
\end{abstract}

European Journal of Endocrinology 148 245-251

\section{Introduction}

The association between type 1 diabetes (T1DM) and autoimmune thyroid disease (AITD) is well established. In many diabetic patients AITD is subclinical and only detected by routine measurements of thyroid autoantibodies (TA) or serum thyrotrophin (s-TSH). The prevalence of TA and thyroid dysfunction increases with age and with duration of diabetes. In children with T1DM cross-sectional studies have shown prevalences of TA between 10 and $22 \%(1-6)$ with a concomitant prevalence of thyroid dysfunction of around $3-7 \%(1,2,4$, 7). In adults with longstanding T1DM $27-44 \%$ of the patients have TA $(8,9)$, while $23-25 \%(9,10)$ have developed thyroid dysfunction (sub- or clinical hypo- or hyperthyroidism).

In a previous population-based, cross-sectional study we investigated children and adolescents with T1DM describing both TA and thyroid ultrasonography (US) as markers of AITD (11). The prevalence of TA was $16 \%$ and clinical or subclinical thyroid dysfunction was found in $5 \%$ of these patients. Additionally, we observed a remarkably high prevalence $(42 \%)$ of US abnormalities (USabn), these being predominantly thyroid hypoechogenicity compatible with AITD (12-14). This finding suggests that in those patients subclinical AITD might be even more common than indicated by the prevalence of TA alone.

Longitudinal studies are needed to clarify the importance of having TA and USabn. Studies in non-diabetics indicate that the risk of developing thyroid dysfunction over time is significantly increased in patients with TA (15) and in patients presenting with thyroid hypoechogenicity (14). In patients with T1DM there are limited data describing the occurrence of TA and the development of thyroid dysfunction in a prospective design $(3,6)$. To our knowledge there are no studies describing the development of thyroid US findings over time. 
The aim of the present study was to investigate the various markers of AITD in young patients with T1DM in a prospective design. The development of thyroid dysfunction, TA and thyroid USabn was evaluated during a 3-year follow-up of the previously investigated cohort.

\section{Subjects and methods}

\section{Subjects}

In 1997 we performed a cross-sectional study of thyroid autoimmunity in $91 \%$ of all young patients with T1DM living in the county of Funen, Denmark (11). A total of 105 diabetics and 105 matched controls, all under 18 years old, were investigated. Three years later, all of the diabetics were offered a follow-up; the re-investigation was performed in $101(96 \%)$ of them (four patients did not want to participate). Among the re-investigated patients, 96 were still living in the county of Funen, four had moved to other parts of Denmark, and one patient was living in the USA but was investigated when she visited Denmark. The patients, 47 girls and 54 boys, had a median age of 15.6 years (range 5.121.1) and a median duration of diabetes of 7.7 years (range 3.2-16.3). Two patients received anticonvulsive treatment, three patients received angiotensin converting enzyme inhibitors because of microalbuminuria, and 11 girls were taking oral contraceptives.

As in the initial investigation (11) all diabetics underwent a physical examination (weight, height, pubertal and goitre status) and a US of the thyroid gland by which the thyroid volume (TV) and morphology were determined. Both the clinical and the US examinations were performed by the same investigators as in the primary study. Venous blood samples were taken in order to determine s-TSH, thyroid hormones, thyroid peroxidase antibodies (TPOab), thyroglobulin antibodies (Tgab) and glycosylated haemoglobin $\left(\mathrm{HbA}_{1 \mathrm{c}}\right)$.

All patients and their parents gave informed consent, and the study was approved by the local ethical committee (case number 20000047). The study was in accordance with the Helsinki Declaration.

\section{Thyroid US}

TV was calculated on the basis of a US scanning procedure employing a $5.5 \mathrm{MHz}$ compound scanner (type 1846, Brüel \& Kjær, Naerum, Denmark) as described previously (16). Two of the authors (F N B and L H) performed all the US investigations without knowledge of the previous US findings. The intra-observer variation using this accurate cross-sectional method, i.e. the coefficient of variation on double determinations, has been assessed previously (17) and was recorded as $6.6 \%(\mathrm{~F} \mathrm{~N} \mathrm{~B})$ and $5.1 \%(\mathrm{~L} \mathrm{H})$, the interobserver variation was $5.0 \%$. In the initial study the echo pattern was investigated with a real-time $7 \mathrm{MHz}$ linear array transducer (type 8534, Brüel \& Kjær, Naerum, Denmark). At the follow-up the echo pattern was investigated using a LOGIQ 500 US scanner (GE Medical Systems, Milwaukee, Wisconsin, USA) with a $12 \mathrm{MHz}$ linear transducer (type 739L). In the present, as well as in the previous setting, the gain was adjusted to produce an echo-free appearance of the lumen of the internal jugular veins, carotid arteries and neckstrap muscles. In both settings a normal thyroid gland has a medium grey scale homogeneous echo pattern and the level of echogenicity is higher than that of the surrounding muscles. The echo pattern was investigated using both transducers for the first 30 patients to calibrate the appearance on the two TV monitors and thus to ensure concordant assessments of the morphology. The different echo patterns observed by US were divided into five groups: (I) normal thyroid gland, (II) mild to moderate diffuse hypoechogenicity, (III) marked diffuse hypoechogenicity, (IV) non-homogeneous hypoechogenicity, and (V) uni- or multinodularity.

\section{Biochemical measurements}

Serum thyroxine $\left(\mathrm{T}_{4}\right)$ (normal range 67-134 nmol/l) and serum tri-iodothyronine $\left(\mathrm{T}_{3}\right)$ (normal range $1.35-2.33 \mathrm{nmol} / \mathrm{l}$ ) was analysed by the fluoroimmunoassay, Auto Delfia (Wallac Oy, Turku, Finland). s-TSH (normal range $0.3-4.0$ arbitrary units/litre $(\mathrm{AU} / \mathrm{l})$ ) was determined by Auto Delfia. Serum TPOab (normal range $<60 \mathrm{U} / \mathrm{ml}$ ) and serum Tgab (normal range $<60 \mathrm{U} / \mathrm{ml}$ ) were determined by RIA (Brahms Diagnostica $\mathrm{GmbH}$, Berlin, Germany). $\mathrm{HbA}_{1 \mathrm{c}}$ (normal range $4.3-6.3 \%$ ) was determined by HPLC.

Subclinical hypothyroidism was defined as an elevated s-TSH level $(>4.0 \mathrm{AU} / \mathrm{l})$ together with normal serum thyroid hormone levels. Overt hypothyroidism was defined as an elevated s-TSH together with a decreased serum thyroid hormone level. Subclinical hyperthyroidism was defined as a decreased s-TSH $(<0.3 \mathrm{AU} / \mathrm{l})$ together with normal thyroid hormone levels. Overt hyperthyroidism was defined as a decreased s-TSH together with elevated thyroid hormone levels.

\section{Statistics}

The results are presented as medians with ranges and as proportions with 95\% confidence intervals (CI). Since data were not normally distributed, nonparametric tests were used in the statistical analyses. For the comparison of paired data, Wilcoxon signed rank sum test and McNemar's tests were used and for the comparison of unpaired data, Wilcoxon, Mann-Whitney and Fisher's exact tests were used. The relationship between TV and other variables was studied by multiple linear regression-analysis. $P$ values of less than 0.05 were considered significant. The STATA statistical package (Intercooled STATA 
7.0, STATA CORP, College Station, TX, USA) was used for the data analyses.

\section{Results}

Clinical and biochemical characteristics from the 101 diabetics who participated in both the initial and the 3-year follow-up study are presented in Table 1.

\section{Thyroid dysfunction}

In the initial study, five patients $(5.0 \%)$ had thyroid dysfunction (two with overt hypothyroidism and three with subclinical hypothyroidism). At follow-up an additional three patients had developed thyroid dysfunction (one with overt hypothyroidism and two with subclinical hypothyroidism), giving an overall thyroid dysfunction prevalence of $7.9 \%\left(\mathrm{CI}_{95 \%}\right.$ : 3.5$15.0 \%$ ). Only one of them had presented symptoms of hypothyroidism before diagnosis. As a group they did not differ significantly to the remaining diabetics with respect to their age, duration of diabetes, age at diabetes onset or metabolic control (Table 2). TPOab were present in four patients, Tgab in three and all had US hypoechogenicity compatible with autoimmune thyroid disease.

\section{Thyroid antibodies}

Among the 17 patients with TA at the initial investigation, antibodies were still present in 13 patients at follow-up. The 13 patients with TPOab at the initial investigation remained positive during follow-up. In most patients the TPO antibody concentration had increased during this period, but in a few it had decreased (Fig. 1). Among 14 patients with Tgab at the initial investigation only seven had Tgab at follow-up. These seven patients were all TPOab positive. None of the initially thyroid antibody-negative patients developed TA during the three-year follow-up. Thus, the overall prevalence of TA was $12.9 \%\left(\mathrm{CI}_{95 \%}: 7.0-\right.$ $21.0 \%)$. There was no significant difference between the prevalence of TA in males $(9.3 \%)$ and females $(17.0 \%)(P=0.372)$. In patients with thyroid dysfunction four of the eight (50\%) had TPOab and three of the eight $(38 \%)$ had Tgab.

Comparing the groups of patients with and without TA, the patients with TA had significantly higher s-TSH levels $(P=0.003)$, while there was no significant difference when comparing $\mathrm{T}_{4}, \mathrm{~T}_{3}, \mathrm{TV}, \mathrm{HbA}_{1 \mathrm{c}}$, age, duration of diabetes or age at diabetes onset (Table 3 ).

\section{Thyroid ultrasonography}

Thyroid US demonstrated a median TV of $10.7 \mathrm{ml}$ (range 3.3-34.5) at follow-up, compared with $8.7 \mathrm{ml}$ (range 1.5-23.2) at the primary investigation $(P<$ 0.001) (Table 1). TV correlated significantly with several variables (age, height, weight, BMI, duration of diabetes, age at diabetes onset and $\mathrm{T}_{3}$ ). However, following multiple linear regression analysis, TV was significantly correlated only with age and weight.

The prevalence of the different morphology patterns found by thyroid US is presented in Table 4 together with the results from the initial investigation. We found that $48.5 \%$ of diabetics had some kind of US abnormality at follow-up compared with $41.6 \%$ of diabetics and $10.0 \%$ of healthy controls at the initial investigation. Thirty-three patients had USabn at both examinations. Sixteen patients with an initially normal thyroid morphology pattern had developed USabn at the follow-up, whereas nine patients with USabn at the first examination had a normal US at follow-up. Most patients with USabn at both examinations showed some degree of hypoechogenicity, but in many patients the degree (mild to marked hypoechogenicity) as well as the pattern (diffuse or nonhomogeneous hypoechogenicity) changed between the two examinations. The frequency of USabn was significantly higher in patients with TA (76.9\%) than

Table 1 Clinical characteristics, biochemical measurements and thyroid ultrasonographic findings at the initial investigation and at follow-up. Results presented as medians and ranges, or as frequencies.

\begin{tabular}{lcc}
\hline & $\begin{array}{c}\text { Initial investigation } \\
(n=101)\end{array}$ & $\begin{array}{c}\text { Follow-up } \\
(n=101)\end{array}$ \\
\hline Age (years) & $12.7(2.0-18.2)$ & $15.6(5.1-21.1)$ \\
Height (cm) & $155.0(85.0-193.0)$ & $165.5(105.0-193.0)$ \\
Weight (kg) & $47.0(11.0-86.0)$ & $61.4(17.1-97.0)$ \\
BMl (kg/m $\left.{ }^{2}\right)$ & $19.2(14.5-29.8)$ & $21.5(14.9-31.5)$ \\
HbA $(\%)$ & $8.0(5.6-15.1)$ & $8.8(5.4-16.3)$ \\
TSH (AU/l) & $1.51(0.59-6.18)$ & $1.53(0.38-61.6)$ \\
Thyroid dysfunction (\%) & $5.0(5 / 101)$ & $7.9(8 / 101)$ \\
TPOab (\%) & $12.9(13 / 101)$ & $12.9(13 / 101)$ \\
Tgab (\%) & $13.9(14 / 101)$ & $7.0(7 / 101)$ \\
Thyroid volume (ml) & $8.7(1.5-23.2)$ & $10.7(3.3-34.5)$ \\
US abnormalities $(\%)$ & $41.6(42 / 101)$ & 4.001 \\
\end{tabular}

*Wilcoxon or McNemar's test. NS, not significant. 
Table 2 Clinical characteristics and biochemical measurements in patients with clinical or subclinical hypothyroidism. Data from both the initial investigation in 1997 and the follow-up investigation in 2000 are presented.

\begin{tabular}{|c|c|c|c|c|c|c|c|c|c|c|c|}
\hline \multirow[b]{2}{*}{ Patient } & \multirow[b]{2}{*}{ Sex } & \multicolumn{2}{|c|}{$\begin{array}{c}\text { Age at } \\
\text { diagnosis } \\
\text { (years) }\end{array}$} & \multicolumn{2}{|c|}{ TSH (AU/I) } & \multicolumn{2}{|c|}{ TPOab (U/ml) } & \multicolumn{2}{|c|}{ Tgab (U/ml) } & \multicolumn{2}{|c|}{$\begin{array}{c}\text { US } \\
\text { (morphology } \\
\text { group) }\end{array}$} \\
\hline & & TIDM & AITD & 1997 & 2000 & 1997 & 2000 & 1997 & 2000 & 1997 & 2000 \\
\hline 1 & $\mathrm{~F}$ & 0.7 & 5.0 & 3.0 & 4.3 & 385 & 1521 & neg & neg & II & II \\
\hline 2 & $\mathrm{~F}$ & 1.0 & 4.7 & 3.3 & 61.6 & 89 & 181 & 77 & 77 & I & III \\
\hline 3 & $\mathrm{~F}$ & 1.9 & 7.9 & 4.5 & * & 194 & $>3000$ & $>2000$ & 62 & II & III \\
\hline 4 & $\mathrm{~F}$ & 3.4 & 18.4 & 1.7 & 4.8 & neg & neg & neg & neg & II & II \\
\hline 5 & $\mathrm{~F}$ & 6.4 & 12.3 & * & $*$ & neg & neg & neg & neg & IV & II \\
\hline 6 & $M$ & 10.5 & 13.0 & 6.2 & 4.1 & neg & neg & 290 & neg & II & II \\
\hline 7 & M & 10.6 & 12.0 & * & $*$ & $>3000$ & $>3000$ & $>2000$ & $>2000$ & II & IV \\
\hline 8 & $\mathrm{~F}$ & 11.0 & 16.4 & 4.7 & * & neg & neg & neg & neg & i & IV \\
\hline
\end{tabular}

* Patients treated with levothyroxine, two of them diagnosed with AITD prior to this study. Morphology groups: I, normal thyroid gland; II, mild to moderate diffuse hypoechogenicity; III, marked hypoechogenicity; IV, non-homogenous hypoechogenicity. neg, negative.

in patients without TA $(44.3 \%)(P=0.038)$ (Table 3$)$. All patients with subclinical or overt hypothyroidism showed US hypoechogenicity. The group of patients with USabn had a significantly higher TSH compared with patients without USabn $(P=0.027)$ (Table 5), while there was no significant difference in the levels of $\mathrm{T}_{4}$ and $\mathrm{T}_{3}$. There was no difference between the two groups regarding their age, duration of diabetes, age at diabetes onset, $\mathrm{HbA}_{1 \mathrm{c}}$ or TV (Table 5). The frequency of USabn was significantly higher in females $(68 \%)$ than in males $(31 \%)(P<0.001)$.
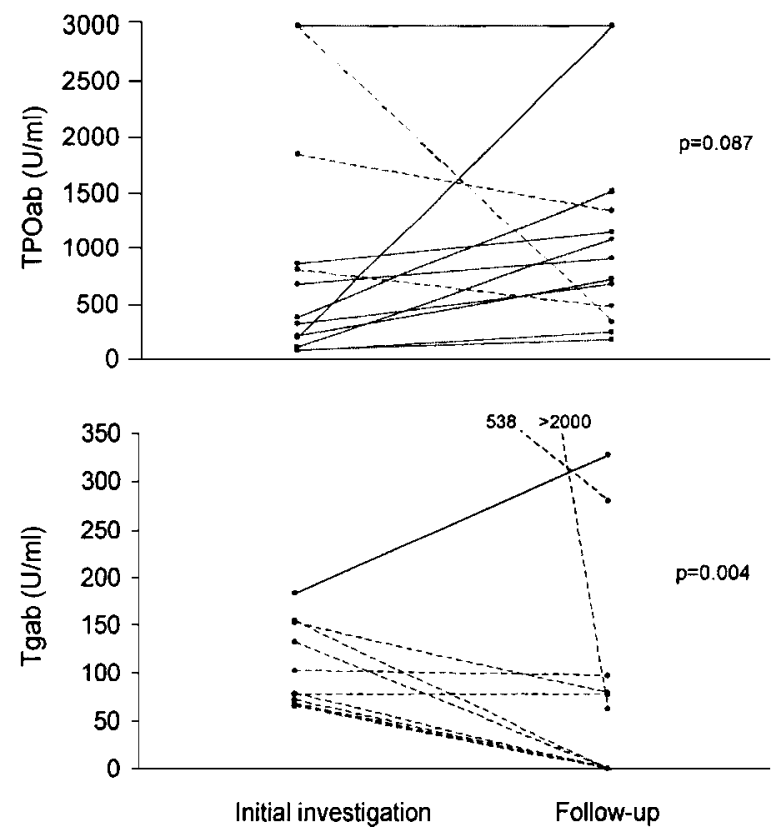

Figure 1 The change in thyroid antibody concentrations during the 3 years between the initial investigation and follow-up. Broken line, patients with a decrease in antibody concentration; unbroken line, patients with an increase in antibody concentration.

\section{Discussion}

During a 3-year follow-up of young patients with T1DM we found an increase in the prevalence of subclinical and overt hypothyroidism from 5 to $8 \%$. Cross-sectional studies in childhood diabetes have demonstrated prevalences of thyroid dysfunction between 3 and $7 \%(1,2$, 4,7 ). Those studies also included children with hyperthyroidism, which was not found in our patients. We expect that the prevalence of thyroid dysfunction in our patients will increase further during the following years and possibly reach the level reported for adult T1DM of $23-25 \%(9,10)$. The question arises of whether it is possible to predict which patients are at an increased risk of developing thyroid dysfunction. TA are known markers of subsequent thyroid dysfunction $(15,18)$. In the present study the prevalence of TPOab was $13 \%$, which remained unaltered during the 3 years of follow-up. It could be speculated that those patients presenting TPOab at both examinations have a higher risk of developing thyroid dysfunction than those who did not. Consistent with this, four of them had developed subclinical or clinical hypothyroidism. The presence of Tgab seems a less reliable marker of subsequent thyroid dysfunction since these antibodies were only found in $7 \%$ of patients, and in seven out of 14 they disappeared by the time of follow-up. This is in agreement with previous studies indicating that Tgab measurements do not contribute to the diagnostic information in AITD (19-21).

The prevalence of TA in our study was $13 \%$ compared with prevalences of $10-22 \%$ in previous studies of children and adolescents with T1DM (1, 4-6). However, in our young patients with a relatively short duration of diabetes, we expect an increasing prevalence of TPOab with time as demonstrated by others $(5,8)$. 
Table 3 Clinical characteristics and biochemical measurements in patients with and without TPOab at the 3-year follow-up investigation. Results presented as medians and ranges, or as a frequency with $95 \%$ confidence intervals.

\begin{tabular}{|c|c|c|c|}
\hline & $\begin{array}{l}\text {-TPOab } \\
(n=88)\end{array}$ & $\begin{array}{c}\text { +TPOab } \\
(n=13)\end{array}$ & $P$ value* \\
\hline Age (years) & $15.2(5.1-21.2)$ & $17.1(5.1-21.1)$ & NS \\
\hline $\operatorname{Sex}(M / F)$ & $49 / 39$ & $5 / 8$ & NS \\
\hline Duration of diabetes (years) & $7.4(3.2-16.3)$ & $8.7(3.3-13.4)$ & NS \\
\hline Age at diabetes onset (years) & $6.5(1.3-16.6)$ & $7.3(0.7-16.5)$ & NS \\
\hline $\mathrm{HbA}_{1 \mathrm{c}}(\%)$ & $8.8(5.4-16.3)$ & $8.7(7.3-15.5)$ & NS \\
\hline TSH (AU/I) & $1.4(0.4-4.8)$ & $2.3(1.1-61.6)$ & 0.003 \\
\hline $\mathrm{T}_{4}(\mathrm{nmol} / \mathrm{l})$ & $110(75-213)$ & $95(9-135)$ & NS \\
\hline $\mathrm{T}_{3}(\mathrm{nmol} / \mathrm{l})$ & $2.2(1.5-3.3)$ & $2.1(1.6-3.1)$ & NS \\
\hline US abnormalities (\%) & $44.3(33.7-55.3)$ & $76.9(46.2-95.0)$ & 0.038 \\
\hline Thyroid volume (ml) & $10.8(3.3-34.5)$ & $10.6(3.9-23.7)$ & NS \\
\hline
\end{tabular}

*Mann-Whitney test or Fisher's exact test. NS, not significant.

Thyroid US is often used in the diagnosis of AITD. In patients with T1DM, the data on US findings are sparse $(2,3,6)$ and no prospective investigation of such patients has to our knowledge been previously reported. Repeating the US examination after 3 years, we found a significant increase in TV, related to and most likely explained by the higher age and weight of our patients at the follow-up examination. Comparing our data with volume data from an extensive investigation of healthy European children and adolescents (22), the TV in the different age groups seems to be higher in diabetic children. However, this may well be explained by differences in urinary iodine excretion, being borderline in our area (23). As demonstrated in Table 3, there was no difference in TV in patients with or without TA, nor was there any difference in TV in patients with or without USabn (Table 5). Thus, TV measurements are of little use in the prediction of AITD.

Employing thyroid US, several studies in nondiabetics have demonstrated a high prevalence of both diffuse and non-homogenous hypoechogenicity in patients with AITD (12-14). In our initial study we demonstrated a significantly higher prevalence of USabn in patients with T1DM compared with healthy controls (11). Three years later we found the same high prevalence of USabn in the diabetics, with 44 of 101 demonstrating varying degrees of thyroid hypoechogenicity, and five of 101 patients presenting unior multinodularity. In many patients the morphology pattern had changed in between the two investigations. In 28 of 44 patients with thyroid hypoechogenicity this was also found at the initial investigation. In some patients the degree and pattern of hypoechogenicity varied between the two investigations. Thyroid hypoechogenicity correlates well with lymphocytic infiltration of the thyroid gland (24), and fluctuations over time in lymphocytic infiltration might explain the variations in hypoechogenicity seen in our patients. In 16 patients with an initially normal thyroid gland, thyroid hypoechogenicity had developed by the time of follow-up. Most of these patients had only a short duration of diabetes at the initial examination (less than 2-3 years) and, since AITD most often develops some years after the onset of T1DM, this might explain why the thyroid hypoechogenicity first appeared at follow-up. The disappearance of the USabn, as demonstrated in nine patients, is harder to explain. Most of those patients showed non-homogeneous hypoechogenicity at the first investigation; a few had discrete, diffuse hypoechogenicity. Only one of them had TPOab (in low concentrations) and all were euthyroid at both investigations. In those patients, the hypoechogenicity found at the first US examination seems to be unspecific and was most likely explained by a high degree of observer variation and not related to thyroid autoimmunity.

To some extent thyroid hypoechogenicity and other AITD markers are related: (i) patients with USabn had a higher prevalence of TPOab than patients without

Table 4 The prevalence of the different morphological patterns found at thyroid ultrasonography both at the initial investigation (diabetics and controls) and at follow-up 3 years later.

\begin{tabular}{lccc}
\hline & \multicolumn{2}{c}{ Initial investigation } & $\begin{array}{c}\text { Follow-up } \\
\text { Diabetics } \\
(n=101)\end{array}$ \\
\cline { 2 - 4 } & $\begin{array}{c}\text { Controls } \\
(n=101)\end{array}$ & $\begin{array}{c}\text { Diabetics } \\
(n=101)\end{array}$ & $\begin{array}{r} \\
\text { Normal thyroid gland (\%) }\end{array}$ \\
Mild-moderate, diffuse hypoechogenicity (\%) & 90.1 & 58.4 & 51.5 \\
Marked, diffuse hypoechogenicity (\%) & 3.0 & 22.8 & 32.7 \\
Non-homogenous hypoechogenicity (\%) & 1.0 & 12.9 & 3.0 \\
Uni- or multinodularity (\%) & 3.0 & 2.0 & 7.9 \\
\hline
\end{tabular}


Table 5 Clinical characteristics and biochemical measurements in patients with and without thyroid ultrasonographic abnormalities at the 3-year follow-up investigation. Results presented as medians and ranges, or as frequencies with $95 \%$ confidence intervals.

\begin{tabular}{|c|c|c|c|}
\hline & $\begin{array}{l}\text { - USabn } \\
(n=52)\end{array}$ & $\begin{array}{c}\text { +USabn } \\
(n=49)\end{array}$ & $P$ value* \\
\hline Age (years) & $15.6(8.8-21.2)$ & $15.6(5.1-21.1)$ & NS \\
\hline $\operatorname{Sex}(M / F)$ & $37 / 15$ & $17 / 32$ & $<0.001$ \\
\hline Duration of diabetes (years) & $7.8(3.3-16.3)$ & $6.9(3.2-15.1)$ & NS \\
\hline Age at diabetes onset (years) & $7.4(1.8-16.6)$ & $5.9(0.7-15.3)$ & NS \\
\hline $\mathrm{HbA}_{1 \mathrm{c}}(\%)$ & $8.8(5.7-13.4)$ & $8.9(5.4-16.3)$ & NS \\
\hline TSH (AU/I) & $1.4(0.4-3.5)$ & $2.1(0.5-61.6)$ & 0.027 \\
\hline $\mathrm{T}_{4}(\mathrm{nmol} / \mathrm{l})$ & $113(75-177)$ & $109.5(9-213)$ & NS \\
\hline $\mathrm{T}_{3}(\mathrm{nmol} / \mathrm{l})$ & $2.2(1.5-3.3)$ & $2.2(1.6-3.3)$ & NS \\
\hline TPOab (\%) & $5.8(1.2-15.9)$ & $20.4(10.2-34.3)$ & 0.038 \\
\hline Thyroid volume (ml) & $11.2(3.3-21.6)$ & $10.6(3.7-34.5)$ & NS \\
\hline
\end{tabular}

* Mann-Whitney test or Fisher's exact test. NS, not significant.

USabn, (ii) in patients with USabn the serum TSH levels were higher than in patients without Usabn, and (iii) all patients with thyroid dysfunction presented some kind of hypoechogenicity. However, the prevalence of thyroid hypoechogenicity was much higher than the reported prevalence of thyroid dysfunction in adulthood, suggesting that the method is not sufficiently specific as a risk marker of subsequent thyroid dysfunction. Alternatively, it could indicate that there are patients with asymptomatic thyroid autoimmunity existing for years without progressing to thyroid dysfunction. This could possibly have been substantiated by fine-needle aspiration biopsy, which was not performed for ethical reasons.

In conclusion, a high risk of developing AITD with thyroid dysfunction in young patients with T1DM may legitimate annual screening of s-TSH. Thyroid US seems to be a sensitive but non-specific method of AITD detection that is not suitable for screening purposes. The use of TA as a screening parameter is also unsuitable since the autoantibodies may persist for many years in the euthyroid patient before causing thyroid dysfunction.

\section{Acknowledgements}

This study was supported by grants from The Clinical Institute of Research, Odense University, The Agnes and Knut Mørk Foundation, The Gerda and Aage Haensch Foundation and The Dagmar Marshall Foundation.

\section{References}

1 McKenna MJ, Herskowitz R \& Wolfsdorf JI. Screening for thyroid disease in children with IDDM. Diabetes Care 199013 801-803.

2 Darendeliler FF, Kadioglu A, Bas F, Bundak R, Gunoz H, Saka N et al. Thyroid ultrasound in IDDM. Journal of Pediatric Endocrinology $1994733-37$.

3 Lorini R, d'Annunzio G, Vitali L \& Scaramuzza A. IDDM and autoimmune thyroid disease in the pediatric age group. Journal of Pediatric Endocrinology and Metabolism 19969 89-94.
4 Roldan MB, Alonso M \& Barrio R. Thyroid autoimmunity in children and adolescents with Type 1 diabetes mellitus. Diabetes Nutrition and Metabolism 199912 27-31.

5 Holl RW, Bohm B, Loos U, Grabert M, Heinze E \& Homoki J. Thyroid autoimmunity in children and adolescents with type 1 diabetes mellitus. Effect of age, gender and HLA type. Hormone Research 199952 113-118.

6 Kordonouri O, Deiss D, Danne T, Dorow A, Bassir C \& GrutersKieslich A. Predictivity of thyroid autoantibodies for the development of thyroid disorders in children and adolescents with Type 1 diabetes. Diabetic Medicine 200219 518-521.

7 Prina-Cerai LM, Weber G, Meschi F, Mora S, Bognetti E, Siragusa V et al. Prevalence of thyroid autoantibodies and thyroid autoimmune disease in diabetic children and adolescents. Diabetes Care $199417782-783$.

8 Chang CC, Huang CN \& Chuang LM. Autoantibodies to thyroid peroxidase in patients with type 1 diabetes in Taiwan. European Journal of Endocrinology 1998139 44-48.

9 McCanlies E, O'Leary LA, Foley TP, Kramer MK, Burke JP, Libman A et al. Hashimoto's thyroiditis and insulin-dependent diabetes mellitus: differences among individuals with and without abnormal thyroid function. Journal of Clinical Endocrinology and Metabolism 199883 1548-1551.

10 Perros P, McCrimmon RJ, Shaw G \& Frier BM. Frequency of thyroid dysfunction in diabetic patients: value of annual screening. Diabetic Medicine $199512622-627$.

11 Hansen D, Bennedbaek FN, Hansen LK, Hoier MM, Jacobsen BB \& Hegedus L. Thyroid function, morphology and autoimmunity in young patients with insulin-dependent diabetes mellitus. European Journal of Endocrinology $1999140512-518$.

12 Gutekunst R, Hafermann W, Mansky T \& Scriba PC. Ultrasonography related to clinical and laboratory findings in lymphocytic thyroiditis. Acta Endocrinologica 1989121 129-135.

13 Nordmeyer JP, Shafeh TA \& Heckmann C. Thyroid sonography in autoimmune thyroiditis. A prospective study on 123 patients. Acta Endocrinologica 1990122 391-395.

14 Marcocci C, Vitti P, Cetani F, Catalano F, Concetti R \& Pinchera A. Thyroid ultrasonography helps to identify patients with diffuse lymphocytic thyroiditis who are prone to develop hypothyroidism. Journal of Clinical Endocrinology and Metabolism $1991 \quad 72$ 209-213.

15 Vanderpump MP, Tunbridge WM, French JM, Appleton D, Bates D, Rodgers $\mathrm{H}$ et al. The incidence of thyroid disorders in the community: a 20-year follow-up of the Whickham Survey. Clinical Endocrinology 199543 55-68.

16 Hegedus L, Perrild H, Poulsen LR, Andersen JR, Holm B, Schnohr $\mathrm{P}$ et al. The determination of thyroid volume by ultrasound and its relationship to body weight, age, and sex in normal subjects. Journal of Clinical Endocrinology and Metabolism $198356260-263$. 
17 Hegedus L, Karstrup S \& Rasmussen N. Evidence of cyclic alterations of thyroid size during the menstrual cycle in healthy women. American Journal of Obstetrics and Gynecology 1986155 $142-145$.

18 Betterle C, Callegari G, Presotto F, Zanette F, Pedini B, Rampazzo T et al. Thyroid autoantibodies: a good marker for the study of symptomless autoimmune thyroiditis. Acta Endocrinologica 1987 $114321-327$.

19 Feldt RU, Hoier MM, Bech K, Blichert TM, Bliddal H, Date J et al. Anti-thyroid peroxidase antibodies in thyroid disorders and nonthyroid autoimmune diseases. Autoimmunity $19919245-254$.

20 Nordyke RA, Gilbert FI Jr, Miyamoto LA \& Fleury KA. The superiority of antimicrosomal over antithyroglobulin antibodies for detecting Hashimoto's thyroiditis. Achives of Internal Medicine $1993153862-865$.

21 Kumar H, Daykin J, Betteridge J, Holder R, Sheppard MC \& Franklyn JA. Prevalence and clinical usefulness of thyroid antibodies in different disease states of the thyroid. Clinical Endocrinology $1999 \mathbf{5 0}$ 679-680.

22 Delange F, Benker G, Caron P, Eber O, Ott W, Peter F et al. Thyroid volume and urinary iodine in European schoolchildren: standardization of values for assessment of iodine deficiency. European Journal of Endocrinology 1997136 180-187.

23 Knudsen N, Bulow I, Jorgensen T, Laurberg P, Ovesen L \& Perrild H. Goitre prevalence and thyroid abnormalities at ultrasonography: a comparative epidemiological study in two regions with slightly different iodine status. Clinical Endocrinology 2000 53 479-485.

24 Hegedus L. Thyroid ultrasound. Endocrinology and Metabolism Clinics of North America 200130 339-360.

Received 26 June 2002

Accepted 3 October 2002 